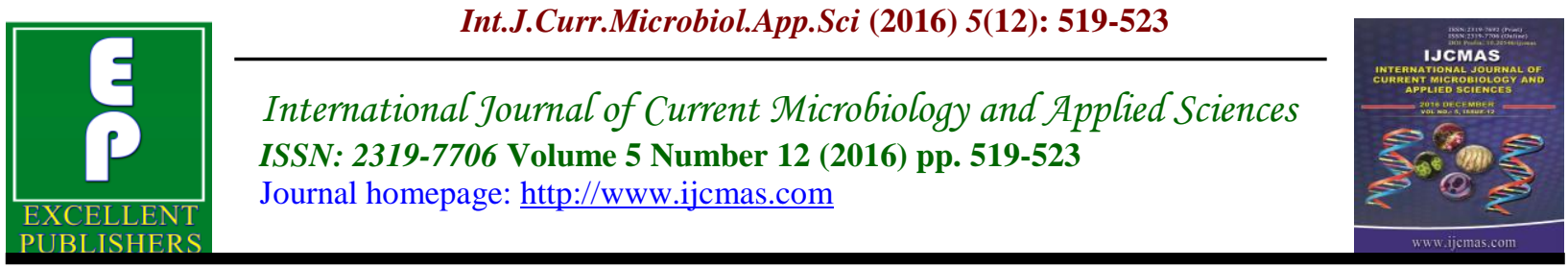

Original Research Article

http://dx.doi.org/10.20546/ijcmas.2016.512.056

\title{
Antimicrobial Sensitivity Pattern of Acinetobacter Species Isolated from Clinical Specimens
}

\author{
Rajani Ranganath ${ }^{1 *}$ and G.S. Vijaykumar ${ }^{2}$ \\ ${ }^{1}$ Department of Microbiology, Raichur Institute of Medical Sciences, Raichur, Karnataka, India \\ 2Department of Microbiology, Shridevi Institute of Medical Sciences, Tumkur, Karnataka, India \\ *Corresponding author
}

Keywords

Acinetobacter, Antibiotic

Resistance.

\section{Article Info}

Accepted:

18 November 2016

Available Online:

10 December 2016

\section{A B S T R A C T}

Acinetobacter species is emerging as a major cause of nosocomial and community acquired infections. To study the resistance pattern of Acinetobacter species. The study was carried out in the Department of Microbiology, Navodaya Medical College, Raichur from December 2013 to November 2014. Out of 5255 clinical samples processed, Acinetobacter species was identified by standard microbiological methods. Modified Kirby Bauer method was used for testing the sensitivity of Acinetobacter species against 11 antimicrobial drugs as per the CLSI guidelines 2011. 72(4\%) revealed Acinetobacter species out of total 1786 (34\%) culture positives. Isolation was maximum in pus (47\%), followed by sputum(17\%), urine(14\%), blood(12\%), CSF(10\%). The sensitivity pattern of Acinetobacter species in this study shows maximum sensitivity to Meropenem (86\%), followed by Piperacillin Tazobactam (76\%), Amiakacin (67\%), Ampicillin Sulbactum(58 $\%)$, Gentamicin (50\%), Ciprofloxacin (49\%), Ceftazidime (40\%), Tetracycline (31\%), Cotrimoxazole (29\%), Ceftriaxone (21\%) and Cefepime (18\%). Development of resistance to commonly used antibiotics has lead to difficulty in treatment of Acinetobacter infections. This type of hospital based data might help to improve the knowledge of antibiotic resistance patterns in this region.

\section{Introduction}

Acinetobacter have been implicated in a wide spectrum of nosocomial and community acquired infections (JollyGuillou, 2005). Emergence of this organism as a significant opportunistic pathogen is because of its ability to survive in any environment and rapid development of resistance to the commonly used antimicrobials (Towner, 1997). Development of resistance in this pathogen is mainly by the production of beta- lactamases and aminoglycoside-modifying enzymes (Robert et al., 2006).

Although it is considered as one of the commensals of the skin and respiratory tract, it can cause a variety of serious infectious diseases like pneumonia, urinary tract infections, endocarditis, wound infections, meningitis, and septicemia (Peleg et al., 2008). Gram negative bacteria are responsible for more than $30 \%$ of hospital 
acquired infections as per the recent data from U.S. National Healthcare Safety Network. Acinetobacter species are the major gram negative bacteria in cases of ventilator -associated pneumonia (47\%) and urinary tract infections (45\%) (Afreenish et al., 2010).

Treatment of infections caused by Acinetobacter species should be based on antibiotic susceptibility pattern because several factors can lead to development of resistance (Halstead et al., 2007; Scott et al., 2007). Very few studies in India have warranted us to undertake this study to report the prevalence of Acinetobacter species, their antibiotic sensitivity pattern and their clinical significance.

\section{Materials and Methods}

This study was conducted in the Department of Microbiology, Navodaya Medical College, Raichur from December 2013 to November 2014. A total of 5255 clinical specimens such as pus, sputum, urine, blood and CSF received from patients admitted to various departments of the Hospital were initially inoculated on Blood agar and MacConkey agar media. Urine samples were inoculated on CLED medium. All isolates obtained were further processed by the routine microbiological and biochemical tests. Typical colonies were subjected to Gram staining, hanging drop, oxidase and catalase test. Acinetobacter was identified on gram staining as gram negative bacilli or coccobacilli, nonmotile, oxidase negative and catalase positive (Mindolli et al., 2010).

Modified Kirby-Bauer disc diffusion method was used for the antibiotic sensitivity testing according to the Clinical and Laboratory Standard Institutes guidelines 2011. The antimicrobial agents used are AmpicillinSulbactum (10 / $10 \mathrm{ug}$ ), Meropenum (10 ug), Gentamycin (10 ug), Ciprofloxacin (5 ug), Pipercillin - tazobactam (100 / $10 \mathrm{ug})$, Amikacin (30 ug), Tetracycline (30 ug), Trimethoprime - sulphamethoxazole (1.25 / $23.75 \mathrm{ug}$ ), Ceftazidime (30 ug), Cefipime (30 ug), Ceftriazone (30 ug) (CLSI, 2011).

\section{Results and Discussion}

Of the total clinical samples processed 1786 (34\%) were culture positive and 3469 (66\%) were culture negative. Overall Acinetobacter species were isolated in $72(45 \%)$ out of 1786 culture positives. The maximum number of Acinetobacter species were isolated from pus (34 out of 72) (47\%), followed by 12 in sputum (17\%), 10 in urine (14\%), 9 in blood (12\%) and least in CSF (7 out of 72$)(10 \%)$ (Table 1)

Chronic obstructive pulmonary disease (COPD), bronchial asthma and respiratory failure were the common respiratory problems in most of our patients. Sensitivity pattern of Acinetobacter species to different antibiotics can be seen in Table 2, which shows most resistant drug was Cefepime $(82 \%)$ and least resistant drug was Meropenem (14\%).

In the present study $72(4 \%)$ isolates of Acinetobacter spp recovered from 1786 positive cultures from different clinical specimens. The results of our study are comparable with Mindolli et al., in Karnataka (4.25\%) and Hisham et al., in Libya (4.2\%) (Mindolli et al., 2010 and Hisham et al., 2012). But higher prevalence rates of $14 \%$ and $9.6 \%$ were observed by Mostofi et al. in Tehran, Iran and Joshi et al. in Pune, India (Mostofi et al., 2011 and Joshi et al., 2006) respectively.

Isolation rate was higher from pus. $12 \%$ were isolated from blood in our study which is slightly higher when compared to those from USA, France, Belgium (7-9.3\%). Studies from various countries have shown 
predominance of isolation from urine (2127\%), tracheobronchial secretions (24.848.8\%) (Lahiri et al., 2004). In our study, Acinetobacter was isolated from urine $(14 \%)$ and sputum $(17 \%)$.

Antibiotic susceptibility pattern of Acinetobacter species against various antibiotics in the present study was maximum with Meropenem (86\%), Piperacillin Tazobactam (76\%), Amiakacin
(67\%), Ampicillin Sulbactum (58\%), Gentamicin (50\%), Ciprofloxacin (49\%), Ceftazidime (40\%), Tetracycline (31\%), Cotrimoxazole (29\%), Ceftriaxone $(21 \%)$ and Cefepime (18\%). In a study conducted by Suri et al. Acinetobacter spp isolated from a neurosurgical unit was sensitive to ciprofloxacin, amikacin, cefotaxim and ceftriaxone (Suri et al., 2000). Singh et al. showed Acinetobacter which was sensitive to amikacin (Singh et al., 2002).

Table.1 Number of Acinetobacter species isolated from various specimens

\begin{tabular}{cc}
\hline Specimens & $\begin{array}{c}\text { No. of Acinetobacter } \\
\text { spp. }\end{array}$ \\
\hline Pus & 34 \\
Sputum & 12 \\
Urine & 10 \\
Blood & 9 \\
CSF & 7 \\
Total & 72 \\
\hline
\end{tabular}

Table.2 Sensitivity pattern of Acinetobacter $\operatorname{spp}(n=72)$ to different antibiotics

\begin{tabular}{lll}
\hline Antibiotics & Sensitive (\%) & $\begin{array}{l}\text { Resistant } \\
(\%)\end{array}$ \\
\hline Ampicillin sulbactam & $42(58)$ & $30(42)$ \\
Meropenem & $62(86)$ & $10(14)$ \\
Gentamicin & $36(50)$ & $36(50)$ \\
Ciprofloxacin & $35(49)$ & $37(51)$ \\
Piperacillin tazobactam & $55(76)$ & $17(24)$ \\
Amikacin & $48(67)$ & $24(33)$ \\
Tetracycline & $22(31)$ & $50(69)$ \\
Cotrimoxazole & $21(29)$ & $51(71)$ \\
Ceftazidime & $29(40)$ & $43(60)$ \\
Cefepime & $13(18)$ & $59(82)$ \\
Ceftriaxone & $15(21)$ & $57(79)$ \\
\hline
\end{tabular}

Prashanth and Badrinath from JIPMER Pondichery isolated Acinetobacter which was sensitive to amikacin and ceftazidime and resistant to ciprofloxacin and cefotaxime (Prashanth et al., 2004). Gladstone et al., from Vellore reported a prevalence of $14 \%$ carbapenem-resistant
Acinetobacter spp., isolated from tracheal aspirates (Gladsone et al., 2005). Prashanth and Badrinath showed gradually increasing resistance of Acinetobacter (Prashanth et al., 2006). As recently as in 2010, one study from Ahmedabad showed few were carbapenem resistant (Patel et al., 2010). 
Some studies have shown higher resistance to carbapenem upto $89 \%$ of isolates (Jaggi et al., 2011). The difference in the sensitivity pattern was due to environmental factors and different patterns of antimicrobial usage.

In conclusion, infections caused by Acinetobacter are difficult to treat these days because of increasing drug resistance. Multifactorial approach consisting of rational use of antibiotics, antibiotic therapy according to the antibiogram results, basic infection control practices and continuous surveillance of antibiotic resistance is the need of the hour.

\section{Acknowledgments}

The authors would like to express their profound gratitude to all the participants for the cooperation and for the immense faith the participants reposed in them.

Conflict of Interest: None to declare.

Ethical Approval: Approval for study was passed from the institutional board of study meeting.

\section{References}

Afreenish, H., Javaid, U., Fatima, K., Aslam, K., Zakir, H. In vitro activity of aminoglycosides, $\quad \beta$-lactam- $\beta$ lactamases inhibitor combinations and tetracyclines against multi-drug resistant Acinetobacter baumannii, isolated from a tertiarycare hospital. $J$. Microbiol. Antimicrob., 4: 47-50.

Clinical and Laboratory Standards Institutes guidelines. 2011.

Gladstone, P., Rajendran, P. and Brahmadathan, K.N. 2005. Incidence of carbapenem resistant nonfermenting gram negative bacilli from patients with respiratory infections in the intensive care units. Indian J. Med. Microbiol., 23: 189191, PMID: 16100428.

Halstead, D.C., Abid, J., Dowzicky, M.J. 2007. Antimicrobial susceptibility among Acinetobacter calcoaceticus-

baumannii complex and Enterobacteriaceae collected as a part of yhe tigecycline evaluation and surveillance trail. J. Infect., 55: 4957. [PubMed: 17250897

Jaggi, N., Sissodia, P. and Sharma, L. 2011. Acinetobacter baumannii isolates: Epidemiology, antibiogram and nosocomial status studied over a 25 month period in a tertiary care hospital in India. Proceedings of the International Conference on Prevention and Infection Control, Geneva, Switzerland.

Jolly-Guillou, M.L. 2005. Clinical impact and pathogenicity of Acinetobacter. Clin. Microbiol. Infect., 11: 868-73.

Joshi, S.G., Litake, G.M., Satpute, M.G., Telang, N.V., Ghole, V.S., Niphadkar, K.B. 2006. Clinical and demographic features of infection caused by Acinetobacter species. Indian $J$. Med. Sci., 60: 351-60. [PubMed: 16940684).

Lahiri, K., Mani, N.S., Purai, S.S. 2004. Acinetobacter species as nosocomial pathogen: Clinical significance and antimicrobial sensitivity. Med. $J$. Armed Forces India, 60: 7-10.

Mindolli, P.B., Salmani, M.P., Vishwanath, G., Hanumanthappa, A.R. 2010. Identification and speciation of Acinetobacter and their antimicrobial susceptibility testing. Al Ameen $J$. Med. Sci., 4: 345-49.

Mostofi, S., Mirnejad, R. and Masjedian, F., 2011. Multi-drug resistance in Acinetobacter baumannii strains isolated from the clinical specimens of three hospitals in Tehran-Iran, 
African J. Microbiol. Res., 26: 44674470.

Patel, M.H., Trivedi, G.R., Patel, S.M. and Vegad, M.M. 2010. Antibiotic susceptibility pattern in urinary isolates of gram negative bacilli with special reference to AmpC $\beta$ lactamase in a tertiary care hospital. Urol. Ann., 2: 7-11. PMID: 20842250.

Peleg, A.Y., Seifert, H., Paterson, D.L. 2008. Acinetobacter baumannii: emergence of a successful pathogen. Clin. Microbiol. Rev., 21: 538-582.

Prashanth, K. and Badrinath, S. 2004. In vitro susceptibility pattern of Acinetobacter species to commonly used cephalosporins, quinolones and aminoglycosides. Indian J. Med. Microbiol., 22: 97-103. PMID: 17642704.

Prashanth, K. and Badrinath, S. 2006. Nosocomial infections due to Acinetobacter species: Clinical findings, risk and prognostic factors. Indian J. Med. Microbiol., 24: 39-44. PMID: 16505554.

Robert, A.B., Dora, S. 2006. Mechanisms of Multidrug Resistance in Acinetobacter species and Pseudomonas aeruginosa. Oxford J., 43: S49-S56, 9.
Scott, P., Deye, G., Srinivasan, A., Murray, C., Moran, K., Hulten, E., et al. 2007. An outbreak of multidrugresistant Acinetobacter baumanniicalcoaceticus complex infection in the US military health care system associated with military operations in Iraq. Clin. Infect. Dis., 44: 157784. [PubMed: 17516401.

Singh, A.K., Sen, M.R., Anupurba, S. and Bhattacharya, P. Antibiotic sensitivity pattern of the bacteria isolated from nosocomial infections in ICU. $J$. Commun. Dis., 34: 257-63. PMID: 14710856.

Suri, A., Mahapatra, A.K. and A. Kapil. 2000. Acinetobacter infection in neurosurgical intensive care patients. Natl. Med. J. India, 13: 296-300. PMID: 11209484.

Towner, K.J. 1997. Clinical importance and antibiotic resistance of Acinetobacter spp. J. Med. Microbiol., 46: 721-746.

Ziglam, H., Elahmer, O., Amri, S., Shareef, F., Grera, A., Labeeb, M., Zorgani, A. 2012. Antimicrobial resistance patterns among Acinetobacter baumannii isolated from burn intensive care unit in tripoli, Libya. Int. Arabic J. Antimicrob. Agents, 2: 3.

\section{How to cite this article:}

Rajani Ranganath and G.S. Vijaykumar. 2016. Antimicrobial Sensitivity Pattern of Acinetobacter Species Isolated from Clinical Specimens. Int.J.Curr.Microbiol.App.Sci. 5(12): 519-523. doi: http://dx.doi.org/10.20546/ijcmas.2016.512.056 